\title{
Restabelecimento estético e funcional de paciente com amelogênese imperfeita utilizando restaurações cerâmicas metal-free
}

\author{
Aesthetic and functional re-establishment of patient with imperfect amelogenesis \\ using metal-free ceramic restorations \\ Restablecimiento estético y funcional del paciente con amelogénesis imperfecta \\ con restauraciones de cerámica sin metal
}

\author{
Danila de OLIVEIRA ${ }^{1}$ \\ Mariana Tassinari CAIXETA ${ }^{\mathbf{1}}$ \\ Fernando Isquierdo de SOUZA ${ }^{2}$ \\ Eduardo Passos ROCHA ${ }^{1}$
}

${ }^{1}$ Departamento de Materiais Odontológicos e Prótese, Faculdade de Odontologia, UNESP, Universidade Estadual Paulista, 16015-050 Araçatuba - SP, Brasil ${ }^{2}$ Curso de Odontologia, Campus Jacarezinho, UENP, Universidade Estadual do Norte do Paraná 86400-000 Jacarezinho - PR, Brasil

\begin{abstract}
Resumo
A amelogênese imperfeita compreende um grupo de condições com alterações do desenvolvimento na estrutura do esmalte dentário que afetam a dentição decídua e permanente e as principais implicações clínicas são: estética prejudicada, hipersensibilidade dentinária, dificuldade na higiene bucal, cáries recorrentes, inflamação gengival e perda da dimensão vertical. O tratamento varia de acordo com o tipo e a gravidade do caso, a fase do desenvolvimento dentário, o nível socioeconômico e as expectativas e anseios do paciente, podendo ser levados anos até o estabelecimento do tratamento definitivo. O presente trabalho tem como objetivo relatar e descrever as etapas clínicas de um caso de amelogênese imperfeita com restabelecimento da estética e função através de laminados cerâmicos ultrafinos, confeccionados em IPS e.max (IvoclarVivadent), com acompanhamento de um ano após a cimentação das restaurações. Conclui-se que os laminados cerâmicos podem ser uma alternativa interessante para o tratamento definitivo de casos mais brandos de amelogênese imperfeita, com otimização dos resultados estéticos e recuperação da autoestima do paciente.
\end{abstract}

Descritores: Amelogênese Imperfeita; Facetas Dentárias; Estética Dentária.

\section{Abstract}

The imperfect amelogenesis comprises a group of conditions with developmental changes in dental enamel structure that affect the deciduous and permanent dentition and the main clinical implications are: impaired esthetics, dentine hypersensitivity, difficulty in oral hygiene, recurrent caries, gingival inflammation and loss of vertical dimension. Treatment varies according to the type and severity of the case, the dental development phase, the socioeconomic level, and the expectations and wishes of the patient, and it may take years to establish definitive treatment. The objective of the present study is to report and describe the clinical stages of a case of imperfect amelogenesis with re-establishment of aesthetics and function through ultrafine ceramic laminates, made of IPS e.max (IvoclarVivadent), followed one year after the cementation of restorations. It is concluded that ceramic laminates can be an interesting alternative for the definitive treatment of milder cases of imperfect amelogenesis, with optimization of aesthetic results and recovery of the patient's self-esteem.

Descriptors: Amelogenesis Imperfecta; Dental Veneers; Esthetics Dental.

\section{Resumen}

La amelogénesis imperfecta comprende un grupo de condiciones con alteraciones del desarrollo en la estructura del esmalte dental que afectan la dentición decidua y permanente y las principales implicaciones clínicas son: estética prejudicada, hipersensibilidad dentinaria, dificultad en la higiene bucal, caries recurrentes, inflamación gingival y pérdida de la enfermedad dimensión vertical. El tratamiento varía de acuerdo con el tipo y la gravedad del caso, la fase del desarrollo dental, el nivel socioeconómico y las expectativas y anhelos del paciente, pudiendo ser llevados años hasta el establecimiento del tratamiento definitivo. El presente trabajo tiene como objetivo relatar y describir las etapas clínicas de un caso de amelogénesis imperfecta con restablecimiento de la estética y función con carillas de cerâmica realizadas con IPS e.max (IvoclarVivadent), con acompañamiento de un año después de la cementación de las restauraciones . Se concluye que las carrillas cerámicas pueden ser una alternativa interesante para el tratamiento definitivo de casos más blandos de amelogénesis imperfecta, con optimización de los resultados estéticos y recuperación de la autoestima del paciente.

Descriptores: Amelogénesis Imperfecta; Coronas con Frente Estético, Estética Dental.

\section{INTRODUÇÃO}

A amelogênese imperfeita compreende um grupo de condições com alterações do desenvolvimento na estrutura do esmalte dentário que afetam a dentição decídua e permanente ${ }^{1,2}$. A etiologia está relacionada com mutação genética durante a fase de formação do esmalte dentário e pode estar associada a uma herança autossômica dominante, autossômica recessiva ou ligada ao cromossomo $\mathrm{X}^{3}$.

A classificação de Witkop, 1988 é a mais usada e divide a amelogênese imperfeita nos padrões: hipoplásico, hipocalcificado, hipomaturado e hipomaturado-hipoplásico ${ }^{4}$. No primeiro, ocorre uma deficiência quantitativa na formação da matriz do esmalte e os dentes envolvidos geralmente são menores em tamanho e apresentam sulcos, fissuras ou áreas sem esmalte dentário ${ }^{4,5}$. Os padrões hipocalcificado e hipomaturado são defeitos qualitativos e em ambos os dentes afetados possuem formato normal ${ }^{6}$. O esmalte hipocalcificado apresenta coloração marrom-amarelado e por ser muito mole, pode ser facilmente perdido ${ }^{4,6}$. O esmalte hipomaturado apresenta manchas opacas de coloração branco-amarelada e/ou amarronzadas e as fraturas e/ou solturas também são comuns neste padrão ${ }^{6,7}$.

As implicações clínicas estão relacionadas principalmente com o tipo e gravidade da amelogênese imperfeita e são elas: estética prejudicada, hipersensibilidade dentinária, dificuldade na higiene bucal, cáries recorrentes, inflamação gengival e perda da dimensão vertical ${ }^{4,8,9}$. Assim como as implicações clínicas, o tratamento varia de acordo com o tipo e a gravidade do caso e, 
também, com a fase do desenvolvimento dentário, o nível socioeconômico e as expectativas e anseios do paciente ${ }^{9,10,11}$. Na dentição decídua e mista os objetivos principais do tratamento são melhorar a estética, diminuir a hipersensibilidade dentinária e evitar a perda do elemento dentário ${ }^{3,8,10}$. Quando estabelecida a dentição permanente, o tratamento reabilitador definitivo pode, então, ser realizado. Sendo as opções de tratamento o cimento de ionômero de vidro, restaurações em resina composta, restaurações cerâmicas e coroas totais metalocerâmicas ou metal-free ${ }^{9,12-14}$. Tratamento ortodôntico e adequação do meio bucal prévios à fase reabilitadora são frequentemente necessários ${ }^{15}$.

O presente trabalho tem como objetivo relatar um caso clínico, com acompanhamento de um ano, de uma paciente com amelogênese imperfeita e o restabelecimento da estética e função através de laminados cerâmicos.

\section{CASO CLÍNICO}

Paciente do sexo feminino, 23 anos, procurou a clínica de pós-graduação da Faculdade de Odontologia de Araçatuba - Unesp, queixando-se de insatisfação estética do seu sorriso e hipersensibilidade dentinária. Durante o primeiro atendimento, foi realizada a anamnese, na qual a paciente relatou diagnóstico prévio de amelogênese imperfeita, que foi confirmado com o exame físico realizado, através da verificação da presença de manchas branco-amareladas/amarronzadas, hipersensibilidade dentinária e facetas de resina composta realizadas devido a fraturas do esmalte dentário (Figuras 1 e 2 ). Foi realizado exame radiográfico a fim de se verificar a existência de cáries e condição da coroa e periodonto dos elementos dentários. Após a conclusão do diagnóstico, foi proposto e aceito como plano de tratamento a confecção de laminados cerâmicos dos elementos 15 a 25 .

Em seguida, foi feita a moldagem diagnóstica com silicone por adição (Express XT, 3M ESPE, St. Paul, MN, USA) e obtenção de dois modelos de estudo com gesso tipo IV (Troquel IV, Polidental, São Paulo, SP, Brasil) os quais foram montados em articulador semi-ajustável (Bio-Art, São Carlos, SP, Brasil) e, juntamente com as fotografias digitais, foram encaminhados ao laboratório de prótese para a confecção do enceramento diagnóstico.

O preparo dental foi baseado no enceramento diagnóstico e guiado por duas máscaras de silicone por condensação (Zetaplus/OranWash, Zhermack, Roma, Italia), sendo uma para orientação no plano vertical e outra no plano horizontal. O desgaste da estrutura dental foi conservador, somente com o objetivo de arredondamento dos ângulos e sem término cervical, com exceção do elemento 21 o qual possuía faceta em resina composta com preparo do término (Figura 3).

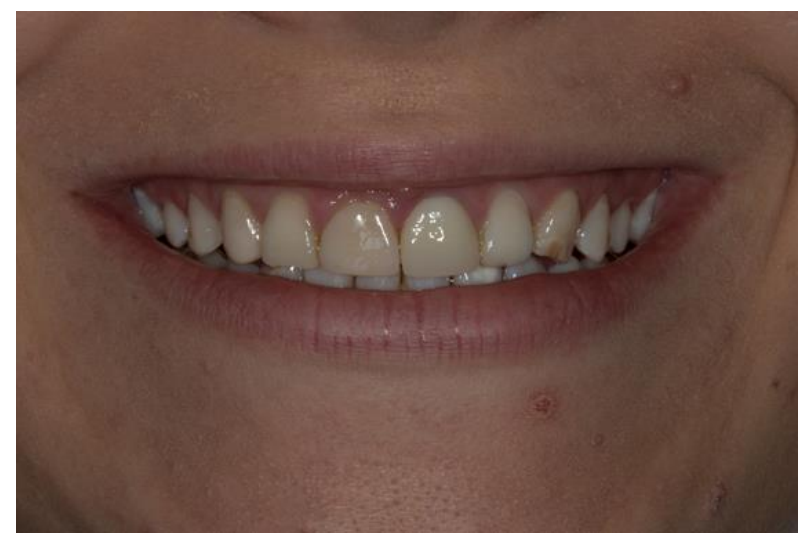

Figura 1: Vista frontal inicial da paciente.

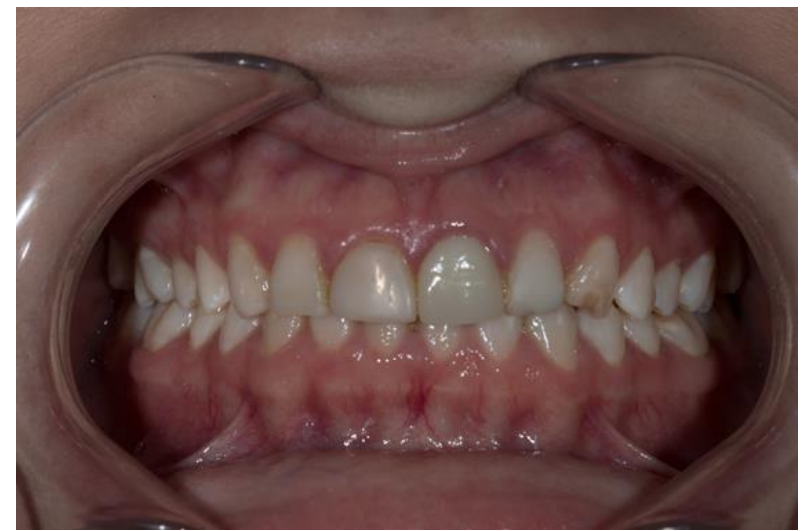

Figura 2: Vista intraoral evidenciando as facetas em resina composta e manchas dentárias características da amelogênese imperfeita.

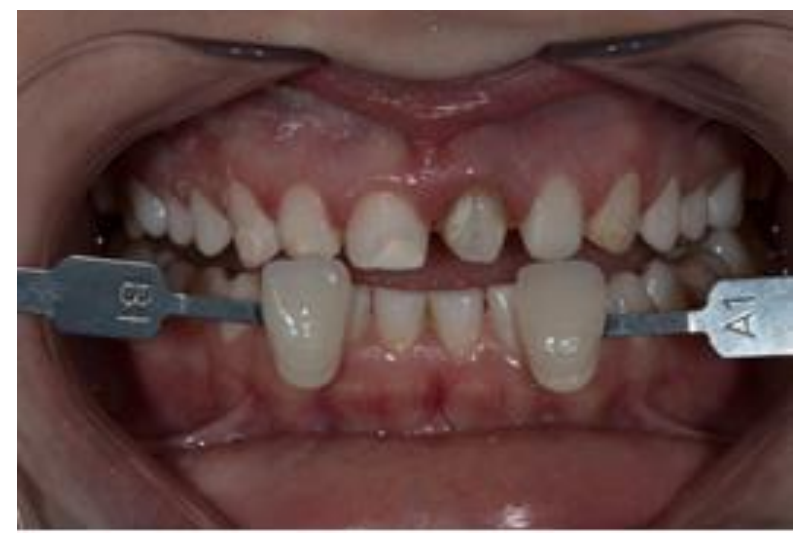

Figura 3: Após a realização dos preparos minimamente invasivos e seleção de cor.

Para o processo de seleção de cor foi utilizada a escala Vita Classical (VITA Zahnfabrik GmbH, Bad Säckingen, Germany), na sequência de luminosidade. Após o preparo, foi realizada a moldagem pela técnica simultânea com duplo fio (Ultrapack, Ultradent Products, South Jordan, Utah, USA), com silicone por adição (Express XT - 3M ESPE, St. Paul, MN, USA). Na sequência foi realizada montagem do modelo de trabalho no articulador semi-ajustável (Bio-Art, São Carlos, SP, Brasil) e registro maxilo-mandibular com material rígido (Occlufast Rock, Zhermack, Roma, Italia). Restaurações provisórias foram confeccionadas com 
resina bis-acrílica (Protemp 4, 3M ESPE, St. Paul, MN, USA) e mantidas até a cimentação dos laminados (Figura 4). As restaurações cerâmicas foram confeccionadas de forma injetada, com o uso do sistema e-Max (IPS e.Max Press, Ivoclar Vivadent, Schaan, Liechtenstein). Na etapa de cimentação, primeiramente realizou a 'prova seca' das restaurações para que fosse visualizado o nível da adaptação de cada peça ao dente. Depois dessa fase, foi realizada a 'prova úmida' com a utilização das pastas de prova (Variolink Esthetic LC Try-in, Ivoclair Vivadent, Schaan, Liechtenstein), para verificação do melhor matiz para cada dente e adequação do nível de mimetização com os dentes naturais de referência. Definida a cor do cimento, realizou o condicionamento das peças com ácido fluorídrico a $10 \%$, ácido fosfórico a $37 \%$ e silano (Monobond Plus, Ivoclair Vivadent, Schaan, Liechtenstein). O condicionamento da estrutura dentária foi feito com ácido fosfórico a $37 \%$ e adesivo (Tetric N-Bond, Ivoclair Vivadent, Schaan, Liechtenstein). Para cimentação foi utilizado cimento resinoso cor Light (Variolink Esthetic LC, Ivoclair Vivadent, Schaan, Liechtenstein) e fotopolimerização de cada face durante 40 segundos (Valo, Ultradent, South Jordan, UT, USA) (Figuras 5 e 6). A paciente possui acompanhamento clínico de 1 ano após a cimentação dos laminados cerâmicos (Figura 7).

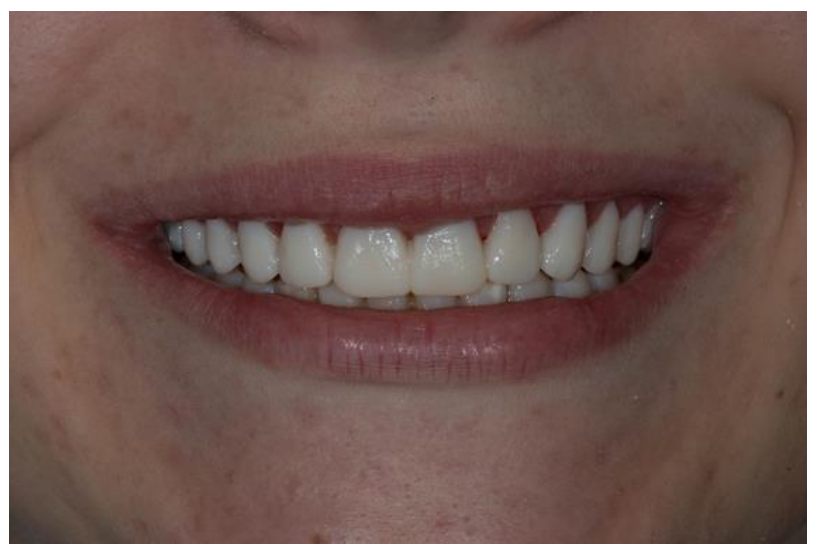

Figura 4: Restaurações provisórias em resina bis-acrílica.

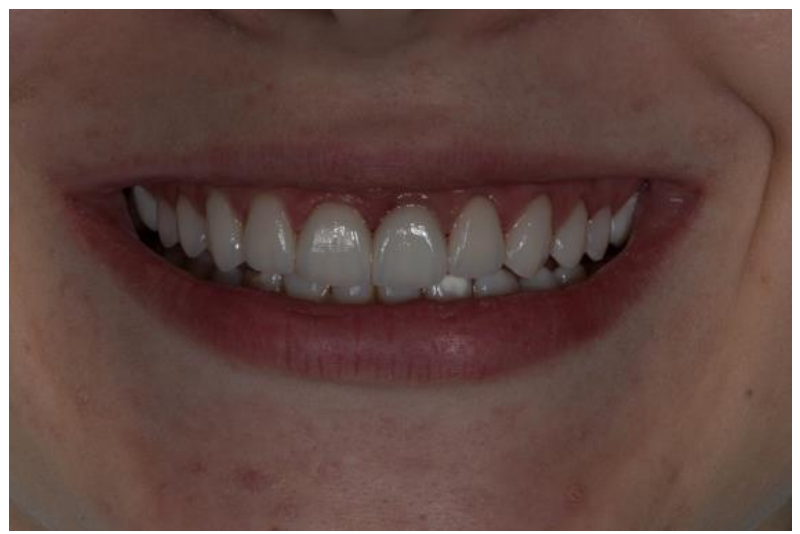

Figura 5: Vista frontal imediatamente após a cimentação das restaurações cerâmicas.

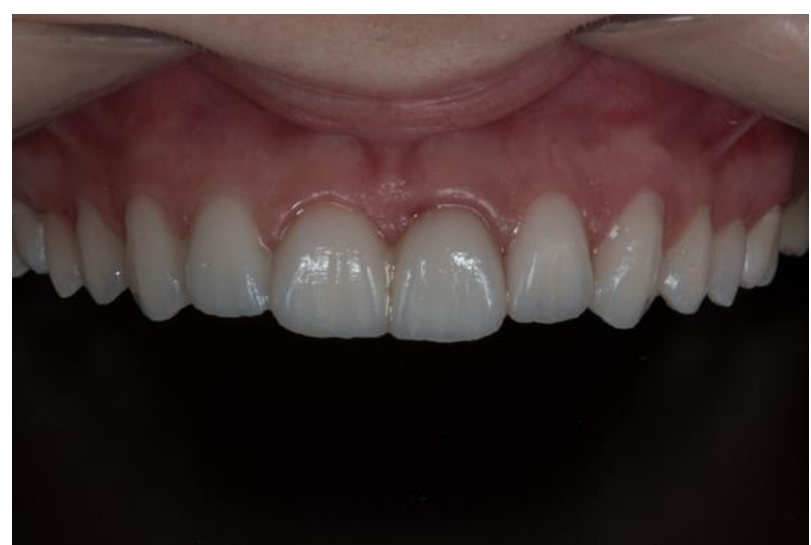

Figura 6: Vista intraoral imediatamente após a cimentação das restaurações cerâmicas.

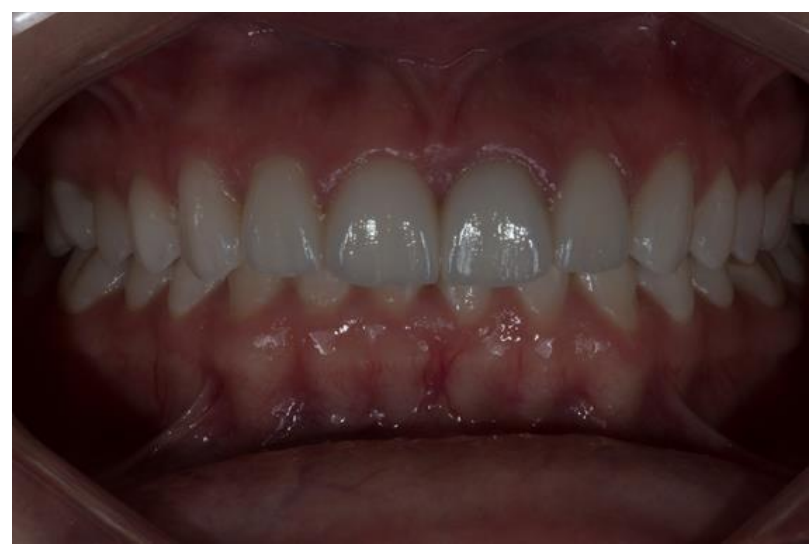

Figura 7: Acompanhamento de 1 ano após a cimentação das restaurações cerâmicas.

\section{DISCUSSÃO}

Estudos publicados na literatura evidenciam vários aspectos negativos da amelogênese imperfeita, tais como: dor e hipersensibilidade dentinária frequentes e acentuadas, dificuldades durante a alimentação, sentimentos de constrangimento e exclusão, baixa autoestima e falta de compreensão de equipes odontológicas sobre o assunto ${ }^{16-18}$, sendo inferior a qualidade de vida relacionada com a saúde quando comparada com indivíduos sem amelogênese imperfeita $^{16,19}$.

O planejamento do tratamento para pacientes com amelogênese imperfeita é complexo e envolve vários fatores ${ }^{10,11}$, tais como um longo período de tempo para o estabelecimento do tratamento definitivo. Antigamente, em casos mais graves, indicavam-se múltiplas extrações dentárias seguida de confecção de próteses totais. ${ }^{20}$ No entanto, atualmente, busca-se a preservação de ambas as dentições através de tratamentos reabilitadores e, devido a evolução dos materiais restauradores, existe uma gama maior de opções, como o cimento de ionômero de vidro, restaurações em resina composta, restaurações cerâmicas e coroas totais metalocerâmicas ou metal-free $e^{9-14}$.

$\mathrm{O}$ restabelecimento da função e da estética com coroas e/ou laminados cerâmicos tem se tornado uma opção de tratamento frequentemente utilizada 
pelos cirurgiões-dentistas ${ }^{9,16,21,22}$. Quando submetidos a esta opção de tratamento, estudos mostram elevadas taxas de satisfação dos pacientes, principalmente quando avaliado o parâmetro "estética"16,19,21,22, como no presente caso ilustrado. Coincidentemente, alguns pacientes relatam que o fator estética é mais relevante que outros, como a hipersensibilidade dentinária e a inflamação gengival ${ }^{16,19}$.

A principal indicação para a indicação de laminados cerâmicos como opção de tratamento da amelogênese imperfeita é quando a demanda estética é superior à gravidade do caso ${ }^{9,11,13}$, ou seja, nos casos mais brandos, como o presente caso ilustrado. E para o sucesso do tratamento alguns critérios devem estar presentes, como, por exemplo, a ausência de problemas periodontais, bom posicionamento dos elementos dentários, bom controle de placa pelo paciente e visitas frequentes ao cirurgião-dentista ${ }^{9,13,19,21}$

\section{CONCLUSÃO}

Os laminados cerâmicos podem ser uma alternativa interessante para o tratamento definitivo de casos mais brandos de amelogênese imperfeita, com otimização dos resultados estéticos e recuperação da autoestima do paciente.

\section{REFERÊNCIAS}

1. Aldred MJ, Savarirayan R, Crawford PJ. Amelogenesis imperfecta: a classification and catalogue for the 21 st century. Oral Dis. 2003;9(1):19-23.

2. Bäckman B, Holm AK. Amelogenesis imperfecta: prevalence and incidence in a northern Swedish county. Community Dent Oral Epidemiol. 1986; 14(1):43-7.

3. Chaudhary M, Dixit S, Singh A, Kunte S. Amelogenesis imperfecta: Report of a case and review of literature. J Oral Maxillofac Pathol. 2009;13(2):70-7.

4. Witkop CJ Jr. Amelogenesis imperfecta, dentinogenesis imperfecta and dentin dysplasia revisited: problems in classification. J Oral Pathol. 1988; 17(9-10):547-53.

5. Ooya K, Nalbandian J, Noikura T. Autosomal recessive rough hypoplastic amelogenesis imperfecta. A case report with clinical, light microscopic, radiographic, and electron microscopic observations. Oral Surg Oral Med Oral Pathol. 1988;65(4):449-58.

6. Witkop CJ Jr, Kuhlmann W, Sauk J. Autosomal recessive pigmented hypomaturation amelogenesis imperfecta. Report of a kindred. Oral Surg Oral Med Oral Pathol. 1973;36(3):367-82.

7. Crawford PJ, Aldred M, Bloch-Zupan A. Amelogenesis imperfecta. Orphanet J Rare Dis. 2007;2:17.
8. Seow WK. Clinical diagnosis and management strategies of amelogenesis imperfectavariants. Pediatr Dent. 1993;15(6):384-93.

9. Yip HK, Smales RJ. Oral rehabilitation of young adults with amelogenesis imperfecta. Int $\mathbf{J}$ Prosthodont. 2003 Jul-Aug; 16(4):345-9.

10.McDonald S, Arkutu N, Malik K, Gadhia K, McKaig S. Managing the paediatric patient with amelogenesis imperfecta. $\mathrm{Br}$ Dent $\mathrm{J}$. 2012;212(9):425-28.

11.Ozturk N, Sari Z, Ozturk B. An interdisciplinary approach for restoring function and esthetics in a patient with amelogenesis imperfecta and malocclusion: a clinical report. J Prosthet Dent. 2004;92(2):112-5.

12.Harley KE, Ibbetson RJ. Dental anomalies--are adhesive castings the solution? $\mathrm{Br}$ Dent $\mathrm{J}$. 1993;174(1):15-22.

13. Walter B. Prosthetic rehabilitation of a case of total amelogenesis imperfecta. Actual Odontostomatol. 1991;45(174):213-26. French.

14.Chen C-F, Hu JCC, Estrella MRP, Peters MC, Bresciani E. Assessment of restorative treatment of patients with amelogenesis imperfecta. Pediatr Dent. 2013;35(4):337-42.

15.Ramos AL, Pascotto RC, Iwaki Filho L, Hayacibara RM, Boselli G. Interdisciplinary treatment for a patient with open-bite malocclusion and amelogenesis imperfecta. Am J Orthod Dentofacial Orthop. 2011;139(4 Suppl):S145-53.

16.Pousette Lundgren G, Wickström A, Hasselblad T, Dahllöf G. Amelogenesis imperfecta and early restorative crown therapy: an interview study with adolescents and young adults on their experiences. PLoS One. 2016;11(6):e0156879.

17. Seymen F, Kiziltan B. Amelogenesis imperfecta: a scanning electron microscopic and histopathologic study. J Clin Pediatr Dent. 2002;26(4):327-35.

18. Gilchrist F, Rodd H, Deery C, Marshman Z. Assessment of the quality of measures of child oral health-related quality of life. BMC Oral Health. 2014;14:40.

19.Pousette Lundgren G, Karsten A, Dahllöf G. Oral health-related quality of life before and after crown therapy in young patients with amelogenesis imperfecta. Health Qual Life Outcomes. 2015;13:197.

20.Toksavul S, Ulusoy M, Türkün M, Kümbüloğlu O. Amelogenesis imperfecta: the multidisciplinary approach. A case report. Quintessence Int. 2004;35(1):11-4.

21.Greenfield R, Iacono V, Zove S, Baer $\mathrm{P}$. Periodontal and prosthodontic treatment of amelogenesis imperfecta: a clinical report. J Prosthet Dent. 1992;68(4):572-74. 
22.Coffield KD, Phillips C, Brady M, Roberts MW, Strauss RP, Wright JT. The psychosocial impact of developmental dental defects in people with hereditary amelogenesis imperfecta. J Am Dent Assoc. 2005;136(5):620-30.

\section{CONFLITO DE INTERESSES}

Os autores declaram não haver conflitos de interesse.

AUTOR PARA CORRESPONDENCIA

Danila de Oliveira

dani-oliveiraa@hotmail.com

Submetido em 19/05/2018

Aceito em 04/10/2018 